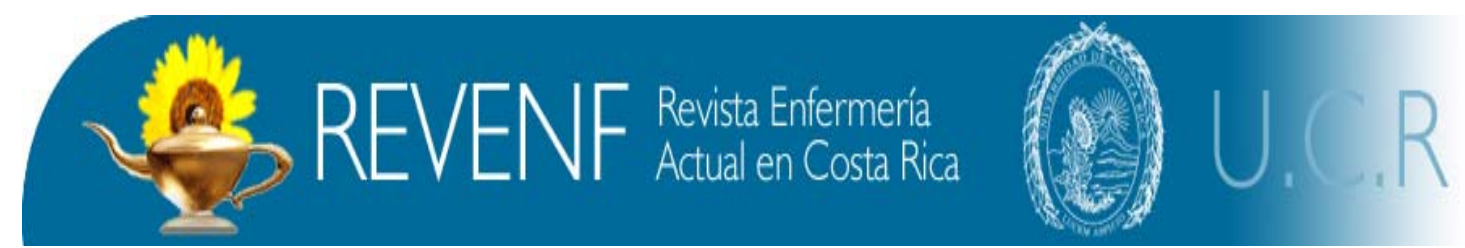

Revista Semestral Número 18 Abril - Setiembre 2010

ISSN 1409-4568

\title{
CONOCIMIENTOS Y PERCEPCIONES DEL PROFESORADO SOBRE VIOLENCIA EN LOS CENTROS EDUCATIVOS PÚBLICOS ${ }^{1}$
}

\section{COMO CITAR ESTE ARTÍCULO}

Alfaro M, Ivannia.; Kenton P., Karen.; Leiva D., Viriam. Conocimientos y percepciones del profesorado sobre violencia en los centros educativos públicos. Rev. Enfermería Actual en Costa Rica [en línea].2010, No.18 [citado (fecha)]. Disponible World Wide Web: <http://www.revenf.ucr.ac.cr/violencia.pdf> ISSN 1409-4568

\author{
Ivannia Alfaro Molina ${ }^{2}$ \\ Karen Kenton Paniagua ${ }^{3}$ \\ Viriam Leiva Díaz ${ }^{4}$
}

Institución: Circuito 03 Dirección Regional de San José.

\begin{abstract}
RESUMEN
Este artículo presenta los resultados de una investigación cuyo objetivo fue explorar los conocimientos y las percepciones que poseen 142 docentes que laboran en las escuelas públicas del circuito 03, de la Dirección Regional de San José, 2008. La investigación fue de tipo descriptiva. Se aplicó un cuestionario autoadministrado con el fin de recolectar la información. Se encontró que la población de docentes no poseen un conocimiento adecuado sobre las causas de la violencia, ni el fenómeno Bullying, desconocen como detectar e intervenir en situaciones de violencia escolar. Además tan solo un $16 \%$ de las personas participantes han recibido algún tipo de capacitación sobre esta temática.
\end{abstract}

Palabras claves: violencia, docentes, capacitación, fenómeno Bullying, escolar, centros educativos.

\footnotetext{
${ }^{1}$ Fecha de recepción: Diciembre 2008

Fecha de aceptación: Marzo 2010

${ }^{2}$ Enfermera, Coopesalud. Correo electrónico: ivaniaalfaro@gmail.com

${ }^{3}$ Enfermera, Coopesalud. Correo electrónico: k.kenton@gmail.com

${ }^{4}$ Enfermera, Psicopedagoga. Profesora Asociada de la Universidad de Costa Rica. Correo electrónico: viriaml@gmail.com
} 


\title{
KNOWLEDGE AND PERCEPTIONS OF TEACHERS ON VIOLENCE IN PUBLIC SCHOOLS
}

\begin{abstract}
This article presents the results of an investigation whose purpose was to explore knowledge and perceptions that have teachers working in 142 public schools in the circuit 03 of the Dirección Regional of San José, 2008. The research was descriptive. A questionnaire was filled out in order to collect information. It was found that the population of teachers lack adequate knowledge about the causes of violence, nor bullying phenomenon, known as detection and intervention in situations of school violence. Furthermore only $16 \%$ of participants had received any training on this topic.
\end{abstract}

Key words: Violence, teachers, Bullying phenomenon, school students, educational center.

\section{INTRODUCCIÓN}

En el año de 1996, la Asamblea Mundial de la Salud declaró que la violencia es un problema de salud pública fundamental y creciente en todo el mundo. La Organización Mundial de Salud (OMS) y la Organización Panamericana de la Salud (OPS), en su primer informe sobre Violencia y Salud (2003), indican que más de 1,6 millones de personas en el mundo pierden la vida y muchas más sufren lesiones no mortales como resultado de la violencia autoinfligida, interpersonal o colectiva. Además, estas organizaciones indican que la violencia en su conjunto es una de las principales causas de muerte en todo el mundo para la población de 15 a 44 años de edad.

Costa Rica no es ajena a esta situación, ya que la violencia es un problema que se presenta en los diferentes ámbitos de la sociedad; es una realidad que encierra al país y que aflige a hombre, mujeres y menores en todos los escenarios en que estos se ubican, incluidos aquellos lugares $o$ centros encargados de promover su desarrollo, como es el caso de los centros educativos.
La escuela es la institución que tiene como fin formar, educar y promover el desarrollo de los niños y las niñas; es la que se encarga de insertar a las nuevas generaciones dentro del entramado social. Sin embargo, esta no se encuentra exenta de los diversos problemas sociales, como la delincuencia, la drogadicción, la pérdida de valores, la corrupción y los alcances de la violencia.

Es evidente, cómo las instituciones responsables en gran parte de la formación académica de los niños y las niñas, se están convirtiendo en un lugar peligroso para ellos, no solo a nivel físico, sino también, emocional, psicológico y social. Por lo tanto, se debe poner atención a estas situaciones, con el fin de velar por el bienestar y la integridad de los y las estudiantes.

Los y las enfermeras, como promotores de la salud, deben tomar un papel activo y determinante en la lucha contra la violencia y llevar a cabo acciones concretas a fin de minimizar esta problemática en el país. Por lo cual esta investigación exploró el conocimiento y 
las percepciones que tiene el personal docente de los centros educativos en materia de violencia, escolar, de tal manera que permita sentar las bases para el planeamiento de propuestas concretas dirigidas a realizar un abordaje efectivo de esta problemática en las escuelas nacionales.

Sustentado en lo anterior se planteó como objetivo general: explorar el conocimiento y percepciones sobre violencia en los centros educativos, que poseen las y los docentes que laboran en escuelas de educación primaria del Circuito 03, de la dirección Regional de San José.

\section{MATERIALES Y MÉTODOS}

El estudio es de tipo descriptivo, ya que la finalidad era registrar, describir $\mathrm{y}$ analizar aspectos de una determinada situación (Hernández, Fernández, Baptista, 2007), en esta investigación se trató de los conocimientos y percepciones que poseían los y las docentes sobre la violencia en las escuelas.

De lo anterior se desprende que la población de esta investigación la constituyo docentes del primer ciclo (de primero a tercer grado) y segundo ciclo (de cuarto a sexto grado), que laboran en las escuelas públicas del Circuito 03 de la Dirección Regional de San José. Se incluyó dentro de esta población únicamente a los maestros y maestras de las materias básicas (Ciencias, Español, Matemáticas y Estudios Sociales).

Los distritos que incluye el Circuito 03 son los siguientes: Zapote, Curridabat, San Francisco de Dos Ríos, Guayabo. La cantidad de profesionales con que cuenta cada una de las escuelas no es homogénea, por lo que se decidió trabajar con toda la población, ya que al extraer una muestra aleatoria, existía la posibilidad de que al contar con pocos docentes en algunos centros educativos no quedaran representados dentro del estudio, por lo que se decidió encuestar a toda la población, que la constituyeron 142 profesionales en educación.

Para la recolección de la información, se aplicó un cuestionario autoadministrado, el cual constó de tres partes: la primera contenía datos sociodemográficos, la segunda indagó el conocimiento sobre violencia en las escuelas, y por último, en la tercera parte se desarrollaba aspectos relacionados con la percepción de las y los docentes sobre violencia.

A fin de probar la validez y confiabilidad del instrumento aplicado, se piloteó el mismo con una muestra de un $5 \%$ que compartía características similares a la población en estudio y que no formaron parte de la misma, esto con la finalidad de buscar coherencia y consistencia en los ítems desarrollados en cada uno de los apartados descritos en el epígrafe previo. El mismo, fue sometido a criterio de jueces, los cuales estuvieron constituidos por un profesional en estadística $\mathrm{y}$ tres docentes en enfermería con experiencia en investigación. Finalizado este proceso se procedió a realizar los cambios pertinentes y aplicarlo a los y las docentes.

\section{RESULTADOS}

A continuación se realizará una breve descripción de la población participante en el estudio.

En cuanto al sexo el $94,4 \%$ de las encuestadas son mujeres. Así mismo, el $55,6 \%$ están casados o casadas; el $22,5 \%$ son solteros o solteras, el $16,2 \%$ están divorciados o divorciadas; el 5,7\% están en unión libre o son viudos o viudas. 
Con respecto al nivel educativo, la totalidad de los y las docentes cuentan con estudios universitarios completos: $7 \%$ tienen un diplomado, el 23\% tienen un bachillerato, un $48 \%$ obtuvieron una licenciatura, un $21 \%$ son magíster, y una persona ostenta el grado académico de doctorado.

En lo concerniente a la edad se encontró que el $44 \%$ de la población se ubicaba entre los 41 y los 50 años, seguido de un $30 \%$ que se encontraba entre los $31 \mathrm{y}$ 40 años.

Asociado a la edad se encontró que un $22 \%$ de los participantes refirieron que habían recibido en algún momento capacitación sobre violencia escolar y de este porcentaje, la mayoría se situaban en el rango de edad de los 31 a 40 años, esto es contrastante con lo que sucede entre el grupo de docentes entre los 41 a 50 años de edad, donde la gran mayoría (82\%) anotaron nunca haber recibido capacitación en esta temática.

De los y las docentes que recibieron capacitación, el 77\% mencionó que esta fue brindada en su lugar de trabajo, un $16 \%$ señalo que fue en la universidad y un $7 \%$ no especifico donde.

En lo concerniente al conocimiento sobre violencia se encontró que un $40 \%$ indicó que el uso del poder siempre genera violencia.

Por otra parte, en lo que respecta a los tipos de violencia, un $59 \%$ de las personas tienen de buen a muy buen conocimiento sobre este tema, el 3,5\% señaló como únicos tipos de violencia la física y la sexual; además, el 10,6\% considera que los insultos, desprecios, burlas o críticas no son formas de violencia, ya que según refirieron no lesionan físicamente a las personas. De igual forma, el $8 \%$ opinan que los pellizcos, las "haladas" de pelo y los empujones son juegos normales que se dan entre los niños y las niñas como parte del proceso de socialización.

Si bien es cierto, el 65\% de la población posee un buen conocimiento sobre las manifestaciones de violencia, llama la atención que el 7,3\% consideran que los apodos, bromas y burlas son parte de los juegos normales en la infancia.

En lo que atañe a las causas de violencia, un $63 \%$ de los y las encuestadas poseen poco conocimiento, por ejemplo, un $37 \%$ manifiestan que algunos individuos desde el nacimiento, tienen una predisposición a ser hostiles, agresivos y violentos.

Otro tanto puede decirse de un 36\% de la población encuestada que manifestó que el ser espectador de actos de violencia, no hace que esta sea vista como algo normal, esta percepción contrasta con el hecho de que un $85 \%$ concordó en que el vivir dentro de un ambiente familiar violento puede provocar que los niños y niñas aprendan conductas violentas como formas de resolución de conflictos, en vez del uso del dialogo y la negociación.

De la misma manera, el $51 \%$ de los maestros y maestras señaló que la aprobación de la violencia por parte de los grupos sociales, no es importante para que esta se produzca $y$ se mantenga.

En lo que concierne al fenómeno Bullying entendido este como "manifestaciones de violencia mantenida, mental o física, guiada por un individuo o por un grupo y dirigida contra otro individuo que no es capaz de defenderse a sí mismo en esa situación, y que se desarrolla en el ámbito escolar" (Cerezo, 2002:133), de los y las encuestadas solo un 6\% conocía sobre 
esta problemática, y de ese porcentaje tan solo una persona poseía un conocimiento adecuado al respecto.

Por otra parte, en lo que se refiere a las consecuencias de la violencia escolar, un $31 \%$ de los participantes manifestaron que la mayor parte de las consecuencias son de orden físico; un 6\% señalo que estas no van más allá de moretones, heridas, rasguños, etc.; en forma similar un $13.2 \%$ no se encuentra de acuerdo en que el ausentismo escolar, las autolesiones y el bajo rendimiento académico, puedan ser indicadores de que el niño o la niña estén viviendo situaciones de violencia escolar.

En lo concerniente al manejo de la violencia, más de la mitad de la población refirió desconocer herramientas que pueden ser utilizadas para el control de la violencia, como es el "Reglamento de evaluación de los aprendizajes” (Ministerio de Educación Pública, 2004) y de los que mencionaron conocerlo, el $83 \%$ considera que este no es efectivo en la práctica, aduciendo que "a veces no se obtiene los resultados deseados”, “el reglamento carece de muchos aspectos a considerar y el docente se encuentra atado de manos", “difícilmente con el instrumento se abarcan correcciones a realizar y por ende no endereza los malos hábitos traídos del hogar”, “el reglamento se limita a rebajar puntos, pero se requiere ayudar y formar al estudiante por medio de otras alternativas”, todas estas manifestaciones apuntan a la necesidad de desarrollar alternativas de formación y programas dentro de los centros educativas para el manejo efectivo y afectivo de la violencia escolar.

Por ende, un $80 \%$ de los participantes indicó que dentro de sus lugares de trabajo no se realizan sesiones para abordar la temática de alternativas de manejo de la violencia escolar; con el agravante de que un $31 \%$ señaló que desconocía la manera en que se debía proceder al presentarse una situación de maltrato en contra del estudiantado, del personal docente o administrativo de la institución.

Como consecuencia de lo anterior, algunos y algunas docentes mencionaron que dentro de las formas más adecuadas para manejar la violencia en las escuelas, están el implantar el miedo, ser imponente, dejar las reglas claras de clase, reprender a los niños y niñas. Vale la pena hacer hincapié que cuatro docentes indicaron estar de acuerdo en el uso de la utilización del castigo físico como método para manejar la problemática en las escuelas.

Ahora bien, en cuanto a la prevención de la violencia, un 91\% de los y las docentes señalo que dentro de sus centros educativos no se cuenta con proyectos dirigidos a prevenir la violencia.

En otro orden de cosas, con respecto a la percepción de la violencia escolar, un $63 \%$ de la población consideró que las situaciones de violencia se presentan con mucha frecuencia en los centros educativos. Ubicándose las agresiones verbales (62\%) como las más frecuentes, seguidas por las agresiones físicas y en último lugar las agresiones sexuales.

Cabe destacar que el $44 \%$ de los y las encuestadas indicó que en algún momento ha realizado algún trato violento en contra del alumnado; además agregado a esto un $37 \%$ señala que dentro de los centros educativos que laboran se presentan situaciones de violencia entre los y las docentes. 
Finalmente, de la población total, un $54 \%$ indicó que considera que el resto de sus compañeros y compañeras ejecutan un mal manejo de los eventos violentos dentro de las aulas.

Una vez presentados los resultados se procede a realizar la discusión final de los resultados.

\section{DISCUSIÓN}

En la población encuestada se evidencia un predominio femenino, lo cual no es de extrañar ya que la enseñanza se ha mantenido mayoritariamente en manos de mujeres. Lo anterior puede estar relacionado con el papel que ha jugado la mujer a través del tiempo en lo que se refiere al cuidado y educación de los niños y niñas, como lo menciona Acker "las aspiraciones de las mujeres y las responsabilidades de su rol familiar, son explicaciones suficientes para dar cuenta de su posición n la enseñanza" (Acker, 2000: 115).Situación que podría justificar que la docencia en educación primaria se concentre mayoritariamente, en manos femeninas.

Si bien es cierto que llama la atención la alta proporción de mujeres respecto de los hombre dentro de la población, no se evidenció diferencias en cuanto al manejo que se da a las situaciones de violencia escolar de parte de los y las docentes; esto es coincidente con lo encontrado por Tamar (2005) quien realizó una investigación sobre el maltrato entre escolares, concluyendo que el sexo del personal docente no incide directamente en la utilización o no de estrategias educativas para la resolución de conflictos entre el alumnado.

En forma similar el estudio mostró que más de la mitad de la población esta casada, hecho que podrí ser un factor positivo y/o protector en la utilización de alternativas para el manejo de las situaciones de violencia en la escuela, pues como lo refiere Tamar (2005) dentro de los resultados obtenidos en la investigación antes mencionada, fue que la maternidad y la paternidad entre el profesorado, representa un factor favorecedor de estrategias resolutivas en los conflictos.

De la misma manera, que el estado civil de la población podría implicar un factor positivo en el manejo adecuado de la violencia escolar, otros factores como el nivel académico, la edad, la capacitación de los y las encuestadas, pueden contribuir positivamente o no en la resolución de problemática de la violencia. De aquí que es digno de mencionar que los y las docentes encuestados poseen un alto grado académico, la mayoría se ubica dentro del rango de edad de 41 a 50 años, ambas cosas implican una mayor experiencia y preparación.

A propósito del epígrafe anterior es digno de notar solo un $16 \%$ del profesorado refirió haber recibió en su formación, contenidos o alguna capacitación en la universidad sobre manejo de la violencia escolar. Esta situación pone en la picota la necesidad de desarrollar en la currícula de la formación tanto de grado como de post grado la temática de la violencia escolar.

Se debe considerar que casi la totalidad de participantes que recibió alguna capacitación sobre violencia en sus centros de trabajo, indicó la necesidad de una mayor formación en el tema, con motivo de esto Tamar encontró que "en la medida que los profesores adquieran habilidades y conocimientos específicos, les permitirá actuar no sólo sobre los típicos o clásicos problemas de disciplina presentes en el contexto "áulico" o fuera de él, sino 
fundamentalmente sobre todos aquellos conflictos que afectan la convivencia escolar alterando de una u otra manera el clima social escolar percibido por cada uno de los agentes educativos (Tamar, 2005:222). Pero para lograr esto es necesario brindar los espacios para que el profesorado adquiera estos conocimientos y habilidades.

La población estudiada mostró tener un conocimiento adecuado sobre los tipos, manifestaciones y consecuencias de la violencia; no fue en lo que respecta a las causas de esta problemática y el fenómeno Bullying, a pesar de la relevancia de ambos temas.

Ahora bien, a pesar de que una gran cantidad de los y las participantes manejan bien la definición de violencia, existe un porcentaje pequeño, que no deja de ser significativo, que asoció la definición de violencia con el uso de poder. Como lo señala el MEP (2004), no es el poder por el poder mismo el que es un elemento negativo, es más bien el uso que se le dé a este lo que ocasiona la violencia; pues como lo apunta Baró (1989), el poder es un elemento que puede llegar a influir en el comportamiento de las personas, positiva o negativamente. Por lo tanto, el profesorado puede influir en el comportamiento del estudiantado convirtiendo el uso del poder en un elemento que repercute positivamente, en la conducta de las y los estudiantes. No obstante, para que el grupo de educadores $\mathrm{y}$ educadoras puedan apropiarse del poder y modificar las conductas del alumnado, se requiere tener claro el concepto de poder y su diferencia con la violencia, así como conocer cuándo y cómo utilizar el poder frente a los niños y niñas, con el objetivo de mantener el orden y la disciplina en la clase.

De igual importancia para el manejo de la violencia es el reconocer que la violencia está relacionada con toda acción capaz de lesionar a un individuo, física, emocional o psicológicamente (OMS;OPS, 2003) (García, Madriaza,2005); ante esto se debe destacar que un porcentaje importante de educadores y educadoras identifican como formas de ésta únicamente la de orden física y sexual; esto es cierto en parte según lo mencionado inicialmente, sin embargo, en el orden práctico puede tener implicaciones negativas, ya que podría llevar a profesorado a no identificar algunas manifestaciones de otros formas de violencia, por no considerarlas como tal, ejemplo de esto es que, algunos y algunas docentes opinan que los insultos, burlas, críticas, pellizcos, empujones, etc., son parte normal de los juegos en la niñez. Por tanto, al no tenerse una noción clara de las implicaciones emocionales que puede generar en los niños y niñas, no se intervenga adecuadamente ante estas situaciones.

Igualmente parte de los participantes mencionaron que el ser espectadores constantes de violencia no hace que esta sea vista como algo natural para las personas que conviven en un ambiente violento, por el contrario como lo señala Martín Baró (2003) la violencia es un constructo de la sociedad, en el que cada orden social establece las condiciones necesarias para que esta se geste y reproduzca; Muñoz, Saavedra y Villalta (2007) agregan además que la violencia no es natural, sino aprendida, por lo que el ser espectadores constantes de ella podría hacer que esta sea vista como algo normal y de lo que no se puede sustraer, evitar. En efecto, Barrantes y Lara agregan que "la agresión y la violencia, son entonces, producto de una reproducción de modelos observados, o de conductas realizadas, reforzadas y estimuladas” (Barrantes y Lara, 2005:43). De ahí que cuando en el ambiente ya sea familiar, escolar o comunal, se producen 
contantes episodios de violencia, estos son introyectados por las personas, sentándose las bases para la construcción y el establecimiento de la violencia como forma de resolución de conflictos. Por tanto, es innegable que al no tener claridad sobre este aspecto los y las docentes no se da una comprensión global de la influencia social sobre la génesis de este problema, $\mathrm{y}$ por tanto, no se procure un ambiente libre de situaciones de violencia dentro del entorno escolar (Montoya, Segura, 2006).

Por otro lado, a pesar de los crecientes índices de la violencia escolar lo que pone sobre el tapete el tema del fenómeno Bullying, la mayor parte de la población encuestada lo desconoce. Aspecto sobre el que se debe prestar atención, ya que no se puede hacer frente a un problema como este, sin un conocimiento adecuado. Benítez, García, y Fernández opinan que "A pesar de la existencia de muchos estudios realizados sobre el fenómeno de "bulling", aún poseemos poca información acerca de cómo los profesores entienden y perciben el fenómeno del maltrato entre sus alumnos. $\mathrm{Y}$ es importante conocerlo porque el profesorado es la piedra angular para la implementación de programas de intervención” (Benítez, García, Fernández, 2007: 153-154). Por tanto, no puede haber una adecuada participación del profesorado en la prevención y manejo del bulling si este no cuenta con el conocimiento necesario para sobre esta temática.

Como se mencionó anteriormente, el bulling se caracteriza por presentarse de manera sostenida en el tiempo y no esporádicamente, con lo cual un o una estudiante puede llegar a convertir a otro en su víctima habitual (Orpinas, Home, 2006). Tener claridad sobre este aspecto es fundamental, pues eso permite saber que quizás la agresión que en determinado momento se presenta en contra de una alumna o alumno, pude que no se trate de un episodio eventual o circunstancial, sino que responda a un maltrato que se ha vendido presentando desde tiempo atrás. Contrariamente, si esto no se conoce, es posible que se dejen pasar por alto muchas agresiones entre estudiantes, pues no se les da importancia, al considerárseles como situaciones esporádicas que no llegan a lesionar seriamente a quienes estén involucrados, concordando con lo manifestado por Prieto, Carrillo, Jiménez (2005).

Cabe agregar que el fenómeno bulling es complejo y en consecuencia, se debe tener conocimiento no solo sobre su concepto, sino, también, sobre las peculiaridades y características presentes en él. Precisamente, en el bulling ocurre una situación interesante: las agresoras $\mathrm{y}$ los agresores generalmente presumen de sentimientos de superioridad, suelen ser populares entre sus compañeros y compañeras $\mathrm{y}$ tienen facilidad para desarrollarse en los deportes y juegos (Fernández, 2007; Olweus, 1998); por tanto, se observa que estos y estas, por lo habitual, no poseen sentimientos de inferioridad. No obstante, más de la mitad del profesorado que mencionaron conocer sobre el fenómeno bulling, indicaron que los y las agresores son poco populares y tienen sentimientos de inferioridad. Asimismo, la mayoría se mostró de acuerdo en que una característica de los agresores y agresoras es la baja autoestima.

Si el personal docente no tiene claridad sobre los aspectos anteriores, podría no detectar correctamente a las partes involucradas dentro del fenómeno bulling, ya sea la víctima, el agresor/ agresora 0 los espectadores y las espectadoras. 
Como lo manifiesta Boggino (2005) si bien es cierto, el cuerpo docente tiene gran responsabilidad sobre la formación de los niños y las niñas, no pueden llevar a cabo esta función si no tienen la preparación necesaria para ello, sobre todo al abordar un problema tan complejo como la violencia. Y con la presente investigación se evidenció que existe un déficit de capacitación para los educadores y las educadoras en lo que respecta a la temática de violencia escolar.

\section{CONCLUSIONES}

Una vez presentado los resultados y el análisis de la información, se procede a presentar las principales conclusiones de la investigación.

1. La mayor parte de las encuestadas y los encuestados nunca había sido capacitada en el tema de la violencia escolar.

2. Además, un porcentaje importante de docentes mencionó que los episodios de violencia se presentaban con gran frecuencia dentro de sus centros educativos, $y$ que a pesar de ello, desconocían la forma en que debería proceder al presentarse este tipo de situaciones contra el estudiantado, personal docente o administrativo.

3. Por lo anterior, se recomienda realizar proyectos de capacitación para el personal docente de las escuelas, sobre la temática de violencia escolar. Además que se elaboren y pongan en práctica propuestas para la prevención y el manejo de la violencia en los centros educativos, más atractivas para el alumnado y personal docente.

4. Asimismo, que se cuente con la participación de enfermeros y enfermeras en los escenarios educativos, que promuevan a través de un enfoque de salud integral, conductas saludables y prácticas de prevención y manejo de la violencia, entre estudiantes y docentes.

\section{BIBLIOGRAFÍA}

Acker, S (2000). Género y Educación: reflexiones sociológicas sobre mujeres, enseñanza y feminismo. Madrid: Ediciones Nancea.

Baró, M. (1989). Sistema, grupos y poder. Psicología social desde Centroamérica II. San Salvador: UCA Editores.

Baró, M. (2003). Poder, ideología y violencia. Madrid: Editorial Trotta.

Barrantes, F. y Lara, D. (2005). Los factores escolares y familiares generadores de violencia en la escuela. Trabajo Final de Graduación para optar por el grado de Licenciatura en Educación Primaria. Universidad de Costa Rica, San José.

Benítez, J., García, A., y Fernández, M. (2007). Conocimiento, percepciones y actitudes hacia el maltrato entre iguales entre el profesorado en activo y los docentes en formación. Psicología Educativa, Vol. 13, pp. 151-167. Recuperado el 1 de julio de 2008, de la base de datos Academic Search Complete.

Boggino, N. (2005). Como prevenir la violencia en la escuela: estudio de casos y orientaciones prácticas. Santa Fe: Homo Sapiens Ediciones.

Cerezo, F. (2002). Conductas agresivas en la edad escolar. Madrid: Ediciones Pirámide.

Fernández, I. (2007). Prevención de la violencia y resolución de conflictos: el clima escolar como factor de calidad. Madrid: Narcea, S.A de Ediciones.

García, M.; Madriaza, P. (2005). Sentido y sinsentido de la violencia escolar: Análisis cualitativo del discurso de estudiantes chilenos. Revista Psykhe. Vol. 14, pp. 21-41.

Hernández, R. Fernández, C. Baptista, P (2007). Metodología de la investigación. 
México D.F: Editorial McGraw-Hill Interamericana.

Ministerio de Educación Pública (2004). Guía metodológica para la formación de niños y niñas como agentes multiplicadores. Comisión de prevención y atención de la violencia, San José, Costa Rica.

Montoya, A.; Segura, S. (2006).Construyendo alternativas par la atención de la violencia en centros educativos. La experiencia en la Escuela Cocorí de Cartago. Tesis para optar por el grado de Licenciatura en Trabajo Social. Universidad de Costa Rica, San José.

Muñoz, M., Saavedra, E., y Villalta, M. (2007). Percepciones y significados sobre la convivencia y violencia escolar de estudiantes de cuarto medio de un liceo municipal de Chile. Revista de Pedagogía. Vol.28, No.82, p.197-224. Recuperado el 10 de octubre de 2008, de http://www.scielo.org.ve/scielo.php?pid= S079897922007000200003\&script=sci_a rttext.
Olweus, D (1998). Conductas de acoso y amenaza entre escolares. Madrid: Ediciones Morata.

OMS, OPS (2003). Informe mundial sobre la violencia y la salud. Publicación Científica y Técnica No. 588. Washington: Autor.

Orpinas, P.; Horne, A. (2006). Bullying prevention: creating a positive school climate and developing social competence. Washington: American Psychological Association.

Prieto, M.; Carrillo, J.; Jiménez, J. (2005) La violencia escolar. Revista Mexicana de Investigación Educativa. Vol. 10, pp. 1027- 1045. Recuperado el 1 de julio del 2008, de la base de datos Academic Search Complete.

Tamar, F. (2005). Maltrato entre escolares (Bullying): Estrategias de manejo que implementan los profesores al interior del establecimiento escolar. Revista Psykhe. Vol. 14, pp. 211-225. 